\title{
PERCEPÇÃO DO DESENVOLVIMENTO DE HABILIDADES PARA VIDA EM JOVENS PRATICANTES DE MODALIDADES ESPORTIVAS COM E SEM DEFICIÊNCIA
}

DOI: 10.22289/2446-922X.V7N1A18

\author{
Gabriel Lucas Morais Freire \\ Lenamar Fiorese \\ Sherdson Emanoel da Silva Xavier ${ }^{1}$ \\ Gustavo Bottega Lunardelli \\ Domingos Rodrigues do Nascimento \\ Marcelen Lopes Ribas \\ José Roberto Andrade do Nascimento Junior
}

\section{RESUMO}

Esta investigação objetivou analisar as percepções de jovens praticantes de modalidades esportivas com e sem deficiência acerca do desenvolvimento de habilidades para vida por meio do esporte, em função do tipo de esporte, sexo, faixa etária e tempo de prática. Participaram da amostra 147 jovens atletas de modalidades olímpicas $(n=57)$ e paralímpicas $(n=90)$, com média de idade de $13.14 \pm 1.37$ anos e tempo de prática de $7.35 \pm 4.83$ anos. Os instrumentos utilizados foram: um questionário sociodemográfico e a Escala de Habilidades para a Vida para o Esporte (PLSSS). A análise de dados foi conduzida por meio dos testes Kolmogorov-Smirnov, $t$ independente e correlação de Pearson $(p<0.05)$. Os praticantes mais experientes obtiveram maiores valores nas dimensões de liderança e comunicação $(p<0.05)$. Na comparação entre os sexos, os meninos obtiveram maior percepção de aprendizagem nas variáveis: estabelecimento de metas, habilidades sociais e habilidades emocionais $(p<0.05)$. Ao comparar as modalidades, os atletas de esportes olímpicos apresentaram diferenças significativas no trabalho em equipe, solução de problemas, habilidades emocionais, liderança, controle de tempo e comunicação $(p<0.05)$. Conclui-se, com base na concepção de jovens atletas que o esporte pode contribuir com a aprendizagem de características positivas, sendo a prática esportiva no longo prazo um dos possíveis fatores que resultam no êxito deste processo.

Palavras-chave: Esporte; Desenvolvimento Positivo; Jovens.

\section{PERCEPTION OF DEVELOPING LIFE SKILLS IN YOUNG PEOPLE PRACTICING SPORTS MODALITIES WITH AND WITHOUT DISABILITIES}

\footnotetext{
${ }^{1}$ Endereço eletrônico de contato: sherdsonemanoel@hotmail.com

Recebido em 09/03/2021. Aprovado pelo conselho editorial para publicação em 09/04/2021.
} 


\section{ABSTRACT}

This investigation aimed to analyze the perceptions of young practitioners of Olympic and Paralympic modalities about the development of life skills through sport, according to sex, age group and time of practice. The sample included 147 young athletes from Olympic $(n=57)$ and Paralympic $(n=90)$ modalities, with an average age of $13.14 \pm 1.37$ years and practice time of $7.35 \pm 4.83$ years. The instruments used were: a sociodemographic questionnaire and the Life Skills Scale for Sport ( $P$ LSSS). Data analysis was performed using the Kolmogorov-Smirnov tests, independent $t$ and Pearson correlation ( $p<0.05$ ). The most experienced practitioners obtained higher values in the dimensions of leadership and communication $(p<0.05)$. When comparing the sexes, the boys obtained a greater perception of learning in the variables: goal setting, social skills and emotional skills $(p<0.05)$. When comparing the modalities, Olympic sports athletes showed significant differences in teamwork, problem solving, emotional skills, leadership, time control and communication $(p<0.05)$. It is concluded, based on the conception of young athletes, that sport can contribute to the learning of positive characteristics, with sports practice in the long run being one of the possible factors that result in the success of this process.

Keywords: Sports; Positive Development; Young.

\section{PERCEPCIÓN DEL DESARROLLO DE HABILIDADES PARA LA VIDA EN JÓVENES QUE PRACTICAN MODALIDADES DEPORTIVAS CON Y SIN DISCAPACIDAD}

\section{RESUMEN}

Esta investigación tuvo como objetivo analizar las percepciones de los jóvenes que practican deporte con y sin discapacidad sobre el desarrollo de habilidades para la vida a través del deporte, en función del tipo de deporte, sexo, grupo de edad y tiempo de práctica. La muestra incluyó a 147 jóvenes deportistas de las modalidades olímpica $(n=57)$ y paralímpica $(n=90)$, con una edad media de 13,14 $\pm 1,37$ años y tiempo de práctica de 7,35 \pm 4,83 años. Los instrumentos utilizados fueron: un cuestionario sociodemográfico y la Escala de Habilidades para la Vida en el Deporte (PLSSS). El análisis de los datos se realizó mediante las pruebas de Kolmogorov-Smirnov, $t$ independiente y correlación de Pearson $(p<0,05)$. Los practicantes más experimentados obtuvieron valores más altos en las dimensiones de liderazgo y comunicación $(p<0.05)$. En la comparación entre sexos, los chicos obtuvieron una mayor percepción de aprendizaje en las variables: establecimiento de metas, habilidades sociales y habilidades emocionales $(p<0,05)$. Al comparar las modalidades, los deportistas olímpicos mostraron diferencias significativas en el trabajo en equipo, resolución de problemas, habilidades emocionales, liderazgo, control del tiempo y comunicación $(p<0,05)$. Se concluye, a partir de la concepción de los jóvenes deportistas, que el deporte puede contribuir al aprendizaje de características positivas, siendo la práctica deportiva a largo plazo uno de los posibles factores que redundan en el éxito de este proceso.

Palabras clave: Deporte; Desarrollo positivo; Joven. 


\section{INTRODUÇÃO}

O contexto esportivo é um dos principais meios que promovem o desenvolvimento das capacidades física, psíquica e social durante a juventude (Camiré, Trudel, \& Forneris, 2012). envolvimento dos jovens dentro deste contexto promove uma série de benefícios, tanto para o seu desenvolvimento humano como para sua continuidade e sucesso em direção a vida adulta. Assim, para a realização das atividades dentro e fora do contexto esportivo, bem como para garantir a transição para a vida adulta de forma saudável, influenciando positivamente na adaptação, saúde física e mental, os adolescentes precisam desenvolver as habilidades para vida (Papalia, 2013).

Hodge e Danish (1999) definem habilidades para vida como como as habilidades necessárias para lidar com as demandas e desafios da vida cotidiana. Exemplos destas habilidades incluem liderança, trabalho em equipe e habilidades sociais. Pesquisas passadas demonstraram que tais habilidades para vida são desenvolvidas através de atividades extracurriculares, incluindo música, drama e contexto esportivo (Larson, 2020). Evidências científicas comprovam que devido às altas taxas de participação e sua natureza interativa, emocional e socialmente envolvida, a prática esportiva pode fomentar a aquisição destas habilidades (Weiss, Kerkoski, Marchi Júnior, \& Cabral Bonin, 2020).

Nesse sentido, a literatura vem demonstrando que a prática esportiva exerce um papel essencial para o desenvolvimento de habilidades para vida nos jovens (Cronin, 2019). Um agregado de evidências vem demonstrando a contribuição da prática esportiva para 0 desenvolvimento de habilidades para vida (Freire et al, 2020; Freire et al, 2021, Mossman \& Cronin, 2019), tanto em modalidades coletivas (Freire et al, 2021, Mossman \& Cronin, 2019), como individuais (Freire et al, 2020). Freire et al (2020) observaram que a prática esportiva parece ser um fator interveniente no desenvolvimento das habilidades para vida, como trabalho em equipe, estabelecimento de metas, liderança e comunicação.

Apesar de vários estudos internacionais e nacionais abordarem o desenvolvimento de habilidades para vida em jovens, evidenciou-se uma escassez de estudos com a população praticantes de modalidades esportivas com deficiência. Desta forma, compreender como o contexto esportivo afeta a percepção de desenvolvimento de habilidades para vida de jovens com deficiência é de extrema importância para buscar o maior desenvolvimento destes jovens. Nesse sentido, tais estudos dentro do contexto brasileiro são fundamentais para observar a importância de programas esportivos na contribuição para o desenvolvimento de habilidades para vida tanto do jovem com deficiência, como sem deficiência, tendo em vista que essas habilidades poderão servir de fatores protetivos no desenvolvimento de jovens e que também são de grande importância em outras fases do desenvolvimento da juventude (Pierce, Gould, \& Camiré 2017, Paína, Peccin, \& da Costa Padovani, 2018; Yazicioglu, Yavuz, Goktepe, \& Tan, 2012). 
Face ao exposto, a prática esportiva é de fundamental importância no desenvolvimento de diversas habilidades para vida que influenciam em aspectos físicos, sociais e morais dentro e fora do contexto esportivo (Vargas, 2018). Nesse sentido, este estudo se torna relevante uma vez que poderá agregar dados que auxiliem o trabalho dos professores, treinadores, psicólogos do esporte e profissionais da área bem como os familiares de jovens envolvidos no ambiente esportivo. Assim, esse estudo teve como objetivo analisar as percepções de jovens praticantes com e sem deficiência acerca do desenvolvimento de habilidades para vida por meio do esporte, em função do tipo de esporte, sexo, faixa etária e tempo de prática.

\section{MATERIAIS E MÉTODOS}

\section{Desenho do estudo}

Trata-se de um estudo descritivo com delineamento transversal e investigação metodológica (Ato, Lopez, \& Benavente, 2013). O estudo foi desenvolvido por meio das diretrizes do Strengthening the Reporting of Observational Studies in Epidemiology (STROBE) para estudos observacionais (Malta, Cardoso, Bastos, Magnanini, \& Silva, 2010).

\section{Participantes}

Participaram deste estudo 147 jovens praticantes de modalidades sem deficiência $(n=57$, sendo 39 meninos e 18 meninas) e com deficiência ( $n=90$, sendo 12 meninos e 78 meninas), com média de idade de $13.14 \pm 1.37$ anos e tempo de prática de $7.35 \pm 4.83$ anos. Os participantes foram selecionados de forma não probabilística e por conveniência. Os critérios de inclusão foram os seguintes: 1) Praticar a modalidade há no mínimo seis meses; e 2) Fazer parte da equipe/clube há no mínimo três meses. Somente participaram da pesquisa os adolescentes que tiveram o Termo de Consentimento Livre e Esclarecido assinado pelos pais/responsáveis e que assinaram o Termo de Assentimento.

\section{Instrumentos}

Questionário sociodemográfico - foi elaborado pelos próprios pesquisadores um questionário com questões sobre a idade, sexo, modalidade praticada e tempo de prática.

Escala de Habilidades para a Vida no Esporte (P-LSSS): Desenvolvida por Cronin e Allen (2017) e validada para o contexto brasileiro por Nascimento Junior et al. (2019), a escala é composta por 43 itens que visam avaliar o desenvolvimento ou não de oito habilidades para a vida, em ambientes de esporte: trabalho em equipe (ex. trabalhar bem dentro de uma equipa / grupo), estabelecimento de metas (ex. definir objetivos desafiadores), habilidades sociais (ex. envolvo-me em atividades de grupo), resolução de problemas e tomada de decisões (ex. penso bem sobre um 
problema), habilidades emocionais (ex. usa as minhas emoções para me manter concentrado), liderança (ex. organizar os membros da equipa/grupo para trabalharem em conjunto), gerenciamento de tempo (ex. gerencio bem o meu tempo) e comunicação (ex. falo claramente com os outros). Os participantes respondem a escala numa escala tipo likert de 5 pontos onde: 1 (Nada), 2 (um pouco), 3 (moderadamente), 4 (bastante), e 5 (extremamente). Os valores do alfa de Cronbach para as oito subescalas variaram de $\alpha=.71 \mathrm{a} \alpha=.89$ para os praticantes de modalidades sem deficiência e de $\alpha=.70$ a $\alpha=.79$ para os praticantes de com deficiência (Hair, Risher, Sarstedt, \& Ringle, 2019).

\section{Procedimentos de coleta de dados}

Os procedimentos adotados nesta pesquisa obedeceram aos critérios da Ética em Pesquisa com Seres Humanos conforme Resolução no. 466/12 do Conselho Nacional de Saúde. O estudo está integrado ao projeto institucional aprovado pelo Comitê de Ética em Pesquisa da Universidade Federal do Vale do São Francisco (Parecer n 1.648.086). Inicialmente foi realizado contato com os professores/treinadores responsáveis pelos projetos para solicitação da autorização para realização das coletas de dados com os jovens. As coletas foram realizadas nos locais de treinamento das atividades esportivas. A aplicação dos questionários foi realizada de forma coletiva, em uma sala privativa, com aproximadamente 15 praticantes de cada vez, com a ausência dos treinadores. Durante o preenchimento dos questionários os avaliadores ficaram presentes na sala e tiraram as possíveis dúvidas dos participantes a respeito dos itens dos questionários e não foi permitida a conversa entre os participantes durante o preenchimento. O preenchimento dos questionários teve duração de aproximadamente 30 minutos.

\section{Análise de dados}

A análise de dados foi realizada através de estatísticas descritivas e inferenciais. A análise preliminar dos dados foi realizada por meio do teste de normalidade de Kolmogorov-Smirnov e o teste de homogeneidade das variâncias de Levene. Como os dados apresentaram distribuição normal, a média e o desvio padrão foram usados para a caracterização dos resultados. Foi utilizado o teste $t$ independente para a comparação das habilidades para vida em função da faixa etária, sexo e tempo de prática. Para a comparação das habilidades para vida em função da faixa etária e do tempo de prática, os praticantes foram divididos em dois grupos de acordo com o processo de "Median Split" conforme a classificação a seguir: 1) Faixa etária: até 13,14 anos e mais de 13,14 anos; e 2) Tempo de prática: até 7,35 anos e mais de 7,35 anos. A verificação do tamanho do efeito para as comparações entre mais de dois grupos foi realizada por meio do d de Cohen (1992): valor de $\mathrm{d}=.20$ representa pequeno tamanho do efeito, $\mathrm{d}=.50$ médio e $\mathrm{d}=.80$ grande. A correlação de Pearson foi usada para investigar a relação entre as variáveis sociodemográficas (idade e tempo 
de prática) e as habilidades para vida. Todas as análises foram conduzidas no software SPSS 22.0, adotando-se um nível de significância de $p<0.05$.

\section{RESULTADOS}

$\mathrm{Na}$ comparação da percepção das oito subescalas e o escore global de habilidades para vida em função do tipo de modalidade (Tabela 1), foi possível observar diferenças significativas entre os grupos nas subescalas trabalho em equipe $(p=.047)$, solução de problemas $(p=.047)$, habilidades emocionais $(p=.009)$, liderança $(p=.004)$, controle do tempo $(p=.023)$, comunicação $(p=.002)$ e escore global $(p=.008)$, demonstrando que os praticantes de modalidades sem deficiência apresentam percepções superiores a respeito do desenvolvimento de habilidades para vida oriundas da prática esportiva.

Tabela 1 - Comparação das dimensões de habilidades para vida dos jovens praticantes de modalidades esportivas em função do tipo de esporte.

\begin{tabular}{|c|c|c|c|c|}
\hline \multirow{3}{*}{ VARIÁVEIS } & \multicolumn{2}{|c|}{ Modalidade } & \multirow{3}{*}{$\mathbf{p}$} & \multirow{3}{*}{ d } \\
\hline & $\begin{array}{l}\text { Sem Deficiência } \\
\qquad(n=57)\end{array}$ & $\begin{array}{l}\text { Com Deficiência } \\
\qquad(\mathrm{n}=90)\end{array}$ & & \\
\hline & $\mathrm{M}(\mathrm{DP})$ & $\mathrm{M}(\mathrm{DP})$ & & \\
\hline Trabalho em Equipe & $3.93(.66)$ & $3.54(1.63)$ & $.047^{\star}$ & .308 \\
\hline Estabelecimento de Metas & $3.78(.76)$ & $3.37(1.73)$ & .053 & .306 \\
\hline Habilidades Sociais & $3.68(.80)$ & $3.34(1.75)$ & .116 & .249 \\
\hline Solução de Problemas & $3.71(.95)$ & $3.27(1.74)$ & $.047^{\star}$ & .313 \\
\hline Habilidades Emocionais & $3.68(.90)$ & $3.10(1.72)$ & $.009^{\star}$ & .422 \\
\hline Liderança & $3.83(1.22)$ & $3.09(1.81)$ & $.004^{*}$ & .479 \\
\hline Controle do tempo & $3.46(.90)$ & $2.94(1.83)$ & $.023^{\star}$ & .360 \\
\hline Comunicação & $3.85(.80)$ & $3.13(1.90)$ & $.002^{*}$ & .493 \\
\hline Escore global & $3.74(.66)$ & $3.05(1.83)$ & $.008^{*}$ & .501 \\
\hline
\end{tabular}

*Diferença significativa - $\mathrm{p}<.05$ - Teste t independente. Nota: $\mathrm{M}=$ média; $\mathrm{DP}=$ Desvio Padrão.

Na comparação da percepção das oito subescalas e o escore global de habilidades para vida em função do sexo (Tabela 2), foi possível observar diferença significativa nas subescalas estabelecimento de metas ( $p=.036)$, habilidades socias $(p=.042)$ e habilidades emocionais $(p=.050)$, demonstrando que os meninos apresentam percepções superiores a respeito do desenvolvimento de habilidades para vida oriundas da prática esportiva. 
Tabela 2 - Comparação das dimensões de habilidade para vida dos jovens praticantes de modalidades esportivas em função do sexo.

\begin{tabular}{|c|c|c|c|c|}
\hline \multirow{3}{*}{ VARIÁVEIS } & \multicolumn{2}{|c|}{ Sexo } & \multirow{3}{*}{$\mathbf{p}$} & \multirow{3}{*}{ d } \\
\hline & $\begin{array}{l}\text { Masculino } \\
(n=31)\end{array}$ & $\begin{array}{c}\text { Feminino } \\
(n=116)\end{array}$ & & \\
\hline & $\mathrm{M}(\mathrm{DP})$ & $\mathrm{M}(\mathrm{DP})$ & & \\
\hline Trabalho em Equipe & $4.06(.98)$ & $3.59(1.42)$ & .090 & .385 \\
\hline Estabelecimento de Metas & $4.01(1.06)$ & $3.40(1.51)$ & $.036^{\star}$ & .467 \\
\hline Habilidades Sociais & $3.87(1.06)$ & $3.37(1.53)$ & $.042^{*}$ & .379 \\
\hline Solução de Problemas & $3.84(1.32)$ & $3.33(1.53)$ & .095 & .356 \\
\hline Habilidades Emocionais & $3.76(1.33)$ & $3.21(1.50)$ & $.050^{*}$ & .387 \\
\hline Liderança & $3.53(1.39)$ & $3.34(1.70)$ & .568 & .122 \\
\hline Controle do tempo & $3.33(1.44)$ & $3.09(1.58)$ & .442 & .158 \\
\hline Comunicação & $3.62(1.60)$ & $3.35(1.61)$ & .417 & .168 \\
\hline Escore global & $3.49(1.50)$ & $3.27(1.53)$ & .495 & .145 \\
\hline
\end{tabular}

*Diferença significativa $-\mathrm{p}<.05-$ Teste $\mathrm{t}$ independente. Nota: $\mathrm{M}=$ média; $\mathrm{DP}=$ Desvio Padrão.

Não foi encontrada diferença significativa ( $p>0.05)$ na comparação das dimensões e do escore global de habilidades para vida de acordo com a faixa etária (Tabela 3), evidenciando que a idade não parece interferir no desenvolvimento de habilidades para vida em jovens praticantes de esportes.

Tabela 3 - Comparação das dimensões de habilidades para vida dos jovens praticantes de modalidades esportiva em função da faixa etária.

\begin{tabular}{lcccc}
\hline \multirow{2}{*}{ VARIÁVEIS } & \multicolumn{2}{c}{ Faixa Etária } & & \\
\cline { 2 - 3 } & $\begin{array}{c}\text { Até 13,14 anos } \\
(\mathbf{n = 1 0 3})\end{array}$ & $\begin{array}{c}\text { Mais de 13,14 } \\
\text { anos (n=44) }\end{array}$ & $\mathbf{p}$ & d \\
\cline { 2 - 3 } & $\mathrm{M}(\mathrm{DP})$ & $\mathrm{M}(\mathrm{DP})$ & & \\
\hline Trabalho em Equipe & $3.33(1.75)$ & $3.84(1.11)$ & .079 & .348 \\
Estabelecimento de Metas & $3.21(1.69)$ & $3.67(1.31)$ & .110 & .304 \\
Habilidades Sociais & $3.25(1.73)$ & $3.57(1.32)$ & .267 & .207 \\
Solução de Problemas & $3.26(1.75)$ & $3.52(1.38)$ & .348 & .164 \\
Habilidades Emocionais & $3.01(1.67)$ & $3.46(1.38)$ & .096 & .293 \\
Liderança & $3.18(1.72)$ & $3.47(1.61)$ & .330 & .174 \\
Controle do tempo & $2.96(1.73)$ & $3.22(1.48)$ & .345 & .161 \\
Comunicação & $3.14(1.82)$ & $3.52(1.50)$ & .195 & .227 \\
\hline
\end{tabular}




\begin{tabular}{lllll}
\hline Escore global & $3.07(1.77)$ & $3.42(1.40)$ & .204 & .219
\end{tabular}

*Diferença significativa - $\mathrm{p}<.05$ - Teste t independente. Nota: $\mathrm{M}=$ média; $\mathrm{DP}=$ Desvio Padrão.

$\mathrm{Na}$ Tabela 4 pode-se observar a comparação das oito subescalas e do escore global de habilidades para vida em função do tempo de prática. Foi encontrada diferença significativa entre os grupos nas subescalas liderança $(p=.042)$ e comunicação $(p=.039)$, evidenciando que os adolescentes com maior tempo de prática apresentaram escores superiores nas respectivas habilidades.

Tabela 4- Comparação das dimensões de habilidade para vida dos jovens praticantes de modalidades esportiva em função do tempo de prática.

\begin{tabular}{lcccc}
\hline \multirow{2}{*}{ VARIÁVEIS } & \multicolumn{2}{c}{ Tempo de Prática } & \\
\cline { 2 - 3 } & $\begin{array}{c}\text { Até 7,35 anos } \\
(\mathbf{n = 8 2})\end{array}$ & $\begin{array}{c}\text { Mais de 7,35 } \\
\text { anos (n=65) }\end{array}$ & p & d \\
\cline { 2 - 3 } Trabalho em Equipe & M (DP) & M (DP) & \\
\cline { 2 - 3 } Estabelecimento de Metas & $3.48(1.59)$ & $3.86(1.11)$ & .114 & .277 \\
Habilidades Sociais & $3.32(1.70)$ & $3.70(1.18)$ & .113 & .259 \\
Solução de Problemas & $3.28(1.69)$ & $3.63(1.23)$ & .157 & .236 \\
Habilidades Emocionais & $3.31(1.71)$ & $3.54(1.30)$ & .367 & .151 \\
Liderança & $3.19(1.67)$ & $3.43(1.31)$ & .336 & .159 \\
Controle do tempo & $3.06(1.80)$ & $3.63(1.47)$ & $.042 *$ & .346 \\
Comunicação & $2.89(1.81)$ & $3.34(1.30)$ & .095 & .285 \\
Escore global & $3.09(1.84)$ & $3.66(1.34)$ & $.039^{*}$ & .354 \\
\hline
\end{tabular}

*Diferença significativa - $\mathrm{p}<.05$ - Teste t independente. Nota: $\mathrm{M}=$ média; DP= Desvio Padrão.

\section{DISCUSSÃO}

A presente investigação teve como objetivo analisar as percepções de jovens praticantes de modalidades esportivas acerca da percepção de aprendizagem de habilidades para vida por meio do esporte, em função do tipo de esporte, sexo, faixa etária e tempo de prática. Os principais resultados da investigação demonstram que os jovens praticantes de esportes sem deficiência apresentaram percepção superior no desenvolvimento de habilidades para vida do que os jovens praticantes com deficiência (Tabela 1). A idade parece não parece interferir na percepção de desenvolvimento de habilidades para vida em jovens (Tabela 3), enquanto o tempo de prática demonstrou que os adolescentes com maior tempo de prática apresentaram percepções superiores 
nas habilidades para vida (Tabela 4). Em relação ao sexo, os meninos apresentam percepções superiores a respeito do desenvolvimento de habilidades para vida quando comparado as meninas (Tabela 2).

Um dos principais achados deste estudo se refere à maior percepção de desenvolvimento de habilidades para vida em jovens praticantes de modalidades esportivas sem deficiência quando comparados a jovens praticantes de modalidades com deficiência (Tabela 1). Tais achados demonstram que a maioria das habilidades para vida está mais evidenciada nos praticantes de modalidades olímpicas. Com isso, pode-se inferir que os programas esportivos olímpicos parecem favorecer o desenvolvimento de habilidades para vida como: trabalho em equipe, solução de problemas, habilidades emocionais, liderança, comunicação levando a esses jovens a agirem mais proatividade em suas demandas diárias dentro e fora do esporte (Holt et al, 2017; Freire et al, 2021; Cronin et al, 2019).

Por outro lado, nossos achados demonstram a necessidade de ampliação de investigações, discussões e capacitações para o desenvolvimento de programas esportivos para jovens com deficiência voltados para o desenvolvimento de habilidades para vida, uma vez que a literatura tem demonstrado que a prática esportiva sistematizada pode trazer benefícios para as pessoas com deficiência em todos os domínios da autoestima, percepção da qualidade de vida, autoeficácia, imagem corporal, capacitação e motivação para o envolvimento contínuo e consequentemente para o desenvolvimento de habilidades para vida para as demandas dentro e fora do contexto esportivo (Holt et al, 2017; Freire et al, 2021; Cronin et al, 2019).

Ao analisar a comparação de habilidades para vida em função do tempo de prática (Tabela 4), observou-se que os jovens praticantes com maior tempo de prática reportaram maior liderança e comunicação. Tais achados evidenciam que as habilidades de liderar o grupo em atividades dentro e fora do contexto esportivo e saber se comunicar-se com os colegas de equipe, com o treinador, árbitros e pessoas do cotidiano parecem sofrer influência do tempo de prática dos jovens. Assim, os achados encontrados no presente estudo vão de encontro aos encontrados na literatura demonstrando que o tempo de prática favorece no desenvolvimento de habilidades para vida de jovens praticantes de esporte (Camiré et al, 2019; Cronin \& Allen, 2018; Freire et al, 2021).

Outro achado do estudo foi a comparação das habilidades para vida em função da faixa etária, indicando que a idade não parece ser um fator interveniente no desenvolvimento das habilidades para vida em adolescentes praticantes de esportes. Tais achados vão de encontro a de uma recente meta análise que demonstrou que a idade não influencia no desenvolvimento de habilidades para vida, mas sim a qualidade do programa em que o jovem está inserido (Ciocanel, Power, Eriksen, \& Gillings, 2017). Por outro lado, Freire et al. (2021) observaram em jovens brasileiros praticantes de futsal que a idade influencia no desenvolvimento de habilidades para vida, sendo os mais velhos os que apresentavam maiores percepções nos domínios de habilidades para

Rev. Psicol Saúde e Debate. Mai., 2021:7(1): 256-267. 
vida. Nessa perspectiva, a presente investigação apresenta achados a respeito da diferença da idade no desenvolvimento de habilidades para vida em jovens praticantes de esportivas com e sem deficiência.

Em relação a percepção de desenvolvimento de habilidades para vida em função do sexo (Tabela 2), pode-se perceber que os meninos têm uma maior percepção de desenvolvimento das habilidades de estabelecimento de metas, habilidades sociais e habilidades emocionais quando comparados às meninas. Assim, percebe-se que os meninos parecem ter mais definido suas metas, bem como a habilidade de lidar com suas emoções e com outras pessoas dentro e fora do contexto esportivo. Vale destacar que os achados aqui encontrados não corroboram com os encontrados na literatura onde demonstram que o sexo não interfere no desenvolvimento de habilidades para vida (Ciocanel et al, 2017, Freire et al, 2019). Freire et al. (2019) observaram em jovens praticantes esportes individuais brasileiros que o sexo não influenciava na percepção de habilidades para vida, assim como a meta-análise de Cioacanel et al. (2017) em que os autores acrescentam que a qualidade do programa em que os jovens estão inseridos e o apoio dos pares sociais (treinadores, pais) são considerados fatores mais relevantes para o desenvolvimento das habilidades para a vida.

Embora os achados sejam de grande contribuição para a literatura, algumas limitações estão presentes. Em primeiro lugar, por se tratar de um estudo transversal, os dados obtidos não permitem gerar uma causalidade entre os dados. Por esses motivos, outras pesquisas, sobretudo ensaios clínicos randomizados, como objetivo de comparar programas esportivos tradicionais com programas esportivos específicos para desenvolvimento de habilidades para vida devem ser realizadas sobre a temática para complementar os achados. Percebe-se também a necessidade de realizar pesquisas qualitativas com a finalidade entender as diferenças na percepção de habilidade para a vida entre os sexo e modalidades com deficiência e sem deficiência. Por fim, é de extrema importância ressaltar que os sujeitos estão em constante desenvolvimento de habilidades para vida não apenas no contexto esportivo, mas também em outras experiências de vida.

\section{CONSIDERAÇÕES FINAIS}

Conclui-se que os jovens praticantes de modalidades olímpicas, do sexo masculino e com maior tempo de prática demonstraram maior percepção de desenvolvimento de habilidades para vida. Do ponto de vista prático, é de fundamental importância que os profissionais envolvidos na área busquem gerar experiências esportivas positivas para aumentar o engajamento bem como a permanência a longo prazo dos adolescentes, uma vez que essas vivências podem potencializar o desenvolvimento das habilidades para a vida nessa fase da vida. Para isso é fundamental que estes profissionais realizem o planejamento esportivo, englobando os aspectos técnicos, táticos e físicos em consonância com o ensino das habilidades para a vida.

Rev. Psicol Saúde e Debate. Mai., 2021:7(1): 256-267. 


\section{REFERÊNCIAS}

Ato, M., Lopez, J. J., \& Benavente, A. (2013). A classification system for research designs in psychology. Anales de

Psicología,29(3),1038-1059. https://doi.org/10.6018/analesps.29.3.178511

Bruner, M. W., Balish, S. M., Forrest, C., Brown, S., Webber, K., Gray, E., ... \& Shields, C. A. (2017). Ties that bond: Youth sport as a vehicle for social identity and positive youth development. Research quarterly for exercise and sport, 88(2), 209-214. https://doi.org/10.1080/02701367.2017.1296100.

Camiré, M., \& Santos, F. (2019). Promovendo o desenvolvimento positivo dos jovens e habilidades para a vida no esporte juvenil: desafios e oportunidades em meio ao aumento da profissionalização. Journal of Sport Pedagogy \& Research , 5 (1), 27-34.

Camiré, M., Trudel, P., \& Forneris, T. (2012). Coaching and transferring life skills: Philosophies and strategies used by model high school coaches. The sport psychologist, 26(2), 243-260. https://doi.org/10.1123/tsp.26.2.243

Ciocanel, O., Power, K., Eriksen, A., \& Gillings, K. (2017). Effectiveness of positive youth development interventions: A meta-analysis of randomized controlled trials. Journal of youth and adolescence, 46(3), 483-504. doi: 10.1111/ j.1467-8624.1997.tb04233.x .

Côté, J., Turnnidge, J., \& Evans, M. B. (2014). The dynamic process of development through sport. Kinesiologia Slovenica, 20(3). DOI:1318-2269

Cronin, L. D., \& Allen, J. (2017). Development and initial validation of the Life Skills Scale for Sport.

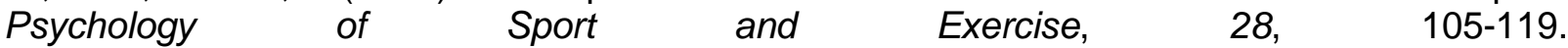
https://doi.org/10.1016/j.psychsport.2016.11.001

Cronin, L. D., \& Allen, J. (2018). Examining the relationships among the coaching climate, life skills development and well-being in sport. International journal of sports science \& coaching, 13(6), 815-827.https://doi.org/10.1177/1747954118787949

Cronin, L., Marchant, D., Allen, J., Mulvenna, C., Cullen, D., Williams, G., \& Ellison, P. (2019). Students' perceptions of autonomy-supportive versus controlling teaching and basic need satisfaction versus frustration in relation to life skills development in PE. Psychology of Sport and Exercise, 44, 79-89.https://doi.org/10.1016/j.psychsport.2019.05.003

Cronin, L., Marchant, D., Allen, J., Mulvenna, C., Cullen, D., Williams, G., \& Ellison, P. (2019). Students' perceptions of autonomy-supportive versus controlling teaching and basic need satisfaction versus frustration in relation to life skills development in PE. Psychology of Sport and Exercise, 44, 79-89.https://doi.org/10.1016/j.psychsport.2019.05.003

Eime, R. M., Young, J. A., Harvey, J. T., Charity, M. J., \& Payne, W. R. (2013). A systematic review of the psychological and social benefits of participation in sport for children and adolescents: informing development of a conceptual model of health through sport. International journal of behavioral nutrition and physical activity, 10(1), 1-21. https://doi.org/10.1186/1479-5868-10-98

Franco, G. D. R., \& Rodrigues, M. C. (2018). Autoeficácia e desenvolvimento positivo dos jovens: Uma revisão narrativa da literatura. Trends in Psychology, 26(4), 22672282.http://dx.doi.org/10.9788/tp2018.4-20pt 
Freire, G. L. M., de Souza Neto, A. C., da Cruz Santos, M., Tavares, J. E. T., de Oliveira, D. V., \& do Nascimento Junior, J. R. A. (2020). Desenvolvimento de habilidades para vida em adolescentes praticantes de esportes individuais. Research, Society and Development, 9(8), e154985557-e154985557.https://doi.org/10.33448/rsd-v9i8.5557

Freire, G. L. M., de Souza Neto, A. C., da Cruz Santos, M., Tavares, J. E. T., de Oliveira, D. V., \& do Nascimento Junior, J. R. A. (2020). Desenvolvimento de habilidades para vida em adolescentes praticantes de esportes individuais. Research, Society and Development, 9(8), e154985557-e154985557.https://doi.org/10.33448/rsd-v9i8.5557

Gomez-Baya, D. (2018, April). Desenvolvimento positivo da juventude e desempenho académico em uma amostra de jovens espanhóis= Positive Youth Development and Academic Performance in a Sample of Spanish Young People. In Congresso Internacional de Psicologia da Criança e do Adolescente (No. 9, pp. 226-227).https://doi.org/10.9788/tp2018.4-20pt

Larson, R. W. (2000). Toward a psychology of positive youth development. American psychologist, 55(1), 170.https://doi.org/10.1037/0003-066X.55.1.170

Malta, M., Cardoso, L. O., Bastos, F. I., Magnanini, M. M. F., \& Silva, C. M. F. P. D. (2010). STROBE initiative: guidelines on reporting observational studies. Revista de saude publica, 44, 559-565. DOI:10.1016/S0025-7753(05)72203-X

Mossman, G. J., \& Cronin, L. D. (2019). Life skills development and enjoyment in youth soccer: The importance of parental behaviours. Journal of sports sciences, 37(8), 850856.https://doi.org/10.1080/02640414.2018.1530580

Nascimento-Junior, J. R. A. D., Fortes, L. D. S., Freire, G. L. M., Oliveira, D. V. D., Fiorese, L., \& Cronin, L. D. (2020). Cross-Cultural Adaptation and Psychometric Properties of the Portuguese Version of the Life Skills Scale for Sport. Measurement in Physical Education and Exercise Science, 24(1), 11-24.https://doi.org/10.1080/1091367X.2019.1647208

Paína, D. M., Fechio, J. J., Peccin, M. S., \& da Costa Padovani, R. (2018). Avaliação da qualidade de vida, estresse, ansiedade e coping de jogadores de futebol de campo da categoria sub-20. Contextos Clínicos, 11(1), 97-105.https://doi.org/10.4013/ctc.2018.111.08

Papalia, D. E., \& Feldman, R. D. (2013). Desenvolvimento humano. Artmed editora. DOI:978-858055-217-1

Pierce, S., Gould, D., \& Camiré, M. (2017). Definition and model of life skills transfer. International review of sport and exercise psychology, 10(1), 186-211. https://doi.org/10.1080/1750984X.2016.1199727

Weiss Ferraz de Oliveira, A., Kerkoski, M. J., Marchi Júnior, W., \& Cabral Bonin Maoski, A. P. (2020). Metodologias facilitadoras na Educação Física: objetivando o desenvolvimento social eo autodomínio emocional. Educación Física y Ciencia, 22.https://doi.org/10.24215/23142561e131

Yazicioglu, K., Yavuz, F., Goktepe, A. S., \& Tan, A. K. (2012). Influence of adapted sports on quality of life and life satisfaction in sport participants and non-sport participants with physical disabilities. Disability and health Journal, 5(4), 249-253. https://doi.org/10.1016/j.dhjo.2012.05.003. 